\title{
Occurrence of Treatment-Related Cardiotoxicity and Its Impact on Outcomes Among Children Treated in the AAML0531 Clinical Trial: A Report
From the Children's Oncology Group
}

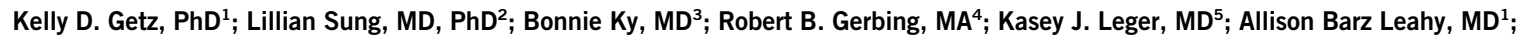
Leah Sack, MS ${ }^{1}$; William G. Woods, MD $^{6}$; Todd Alonzo ${ }^{7}$; Alan Gamis, MD ${ }^{8}$; and Richard Aplenc, MD ${ }^{1}$

PURPOSE Late cardiotoxicity after pediatric acute myeloid leukemia therapy causes substantial morbidity and mortality. The impact of early-onset cardiotoxicity on treatment outcomes is less well understood. Thus, we evaluated the risk factors for incident early cardiotoxicity and the impacts of cardiotoxicity on event-free survival (EFS) and overall survival (OS).

METHODS Cardiotoxicity was ascertained through adverse event monitoring over the course of follow-up among 1,022 pediatric patients with acute myeloid leukemia treated in the Children's Oncology Group trial AAML0531. It was defined as grade 2 or higher left ventricular systolic dysfunction on the basis of Common Terminology Criteria for Adverse Events (version 3) definitions.

RESULTS Approximately $12 \%$ of patients experienced cardiotoxicity over a 5 -year follow-up, with more than $70 \%$ of incident events occurring during on-protocol therapy. Documented cardiotoxicity during on-protocol therapy was significantly associated with subsequent off-protocol toxicity. Overall, the incidence was higher among noninfants and black patients, and in the setting of a bloodstream infection. Both EFS (hazard ratio [HR], 1.6; $95 \% \mathrm{Cl}, 1.2$ to $2.1 ; P=.004$ ) and $\mathrm{OS}(\mathrm{HR}, 1.6 ; 95 \% \mathrm{Cl}, 1.2$ to $2.2, P=.005)$ were significantly worse in patients with documented cardiotoxicity. Impacts on EFS were equivalent whether the incident cardiotoxicity event occurred in the absence ( $\mathrm{HR}, 1.6 ; 95 \% \mathrm{Cl}, 1.1$ to $2.2 ; P=.017)$ or presence of infection ( $\mathrm{HR}, 1.6 ; 95 \% \mathrm{Cl}, 1.0$ to 2.7; $P=.069$ ) compared with patients without documented cardiotoxicity. However, the reduction in OS was more pronounced for cardiotoxicity not associated with infection (HR, $1.7 ; 95 \% \mathrm{Cl}, 1.2$ to $2.5 ; P=.004)$ than for infection-associated cardiotoxicity ( $\mathrm{HR}, 1.3 ; 95 \% \mathrm{Cl}, 0.7$ to $2.4 ; P=.387$ ).

CONCLUSION Early treatment-related cardiotoxicity may be associated with decreased EFS and OS. Cardioprotective strategies are urgently needed to improve relapse risk and both short- and long-term mortality outcomes.

J Clin Oncol 37:12-21. @ 2018 by American Society of Clinical Oncology

\section{INTRODUCTION}

Over the past several decades, cooperative oncology group trials for pediatric acute myeloid leukemia (AML)

See accompanying Oncology Grand Rounds on page 1

Data Supplement

Author affiliations

and support information (if applicable) appear at the end of this article.

Accepted on August 23, 2018 and published at jco.org on October 31, 2018: DOI https://doi.org/10. 1200/JC0.18.00313 previous studies have not evaluated the independent effects of early-onset cardiotoxicity on event-free survival (EFS) or OS in newly diagnosed pediatric patients with AML.

Bacterial sepsis, a cause of transient cardiac decline, occurs frequently during AML treatment and contributes to the development of cardiac dysfunction. ${ }^{16}$ Children's Oncology Group (COG) guidelines for rechallenging patients to anthracyclines distinguish infection-associated LVSD from LVSD not associated with infection, allowing patients who experience resolution of infection-associated LVSD to receive additional anthracycline therapy. However, there are no data comparing treatment outcomes after these distinct presentations of cardiotoxicity.

The primary objectives of this study were to (1) describe the incidence of cardiotoxicity among pediatric patients with AML, (2) assess differences in risk factors 
for incident cardiotoxicity occurring during the on-protocol and off-protocol periods and in the context of infection, and (3) evaluate the impact of cardiotoxicity on EFS and OS. Secondary analyses explored predictors of compliance with routine echocardiographic (echo) monitoring.

\section{METHODS}

\section{Study Population}

Between August 2006 and June 2010, COG AAML0531 enrolled patients younger than 30 years of age for frontline AML treatment as previously described (Data Supplement). ${ }^{2}$ Enrollment criteria did not include baseline ejection fraction (EF) or shortening fraction (SF) cutoffs. Daunorubicin ( $50 \mathrm{mg} / \mathrm{m}^{2} /$ dose or $1.67 \mathrm{mg} / \mathrm{kg} /$ dose if body-surface area $\left.[\mathrm{BSA}]<0.6 \mathrm{~m}^{2}\right)$ and mitoxantrone $\left(12 \mathrm{mg} / \mathrm{m}^{2} /\right.$ dose or $0.4 \mathrm{mg} / \mathrm{kg} /$ dose if $\mathrm{BSA}<0.6 \mathrm{~m}^{2}$ ) were administered as 6-hour intravenous infusions on days 1,3 , and 5 of induction I and induction II, and 1-hour infusions on days 3 to 6 of intensification II, respectively. All patients enrolled in AAML0531 provided informed consent for use of trial data for research. The current analyses excluded patients with Down syndrome.

\section{Cardiotoxicity Ascertainment}

Echo or multigated acquisition (MUGA) scans were mandated at the following times: before the start of induction courses I and II and intensification II, before hematopoietic stem-cell transplantation, at the end of protocol therapy, and at yearly intervals during off-protocol follow-up. As part of routine adverse event (AE) monitoring, cardiac events, regardless of grade, were required to be reported via $A E$ case report forms (CRFs; passive ascertainment). Additional active ascertainment involved targeted requests by the study chair for facsimiles of all echo/MUGA reports for patients with a CRF documenting cardiotoxicity. All EF and SF measurements were abstracted from the submitted echo reports. Discrepancies in LVSD grading between EF mode-specific measurements from the echo report were reviewed and adjudicated by a cardiologist (B.K.).

\section{Outcomes}

Primary outcomes included incident cardiotoxicity, EFS, and OS. Secondary outcomes included compliance with echo monitoring, relapse risk (RR), and nonrelapse or noninduction failure-related mortality (NRM).

The AAML0531 protocol mandated that anthracyclines be withheld if there was evidence of significant cardiac disease, defined as SF $<27 \%$. $^{2}$ Thus, our definition of cardiotoxicity, on the basis of the National Cancer Institute Common Terminology Criteria for Adverse Events (version 3.0), included LVSD grades that would wholly contain patients meeting the protocol-defined threshold for dose modification, specifically grade 2 or higher LVSD (resting $\mathrm{SF}<24 \%$ or $\mathrm{EF}<50 \%$ ). Patients were considered to have experienced LVSD if reported on a CRF or submitted echo report. LVSD occurrences were subclassified as associated with a bloodstream infection or not, on the basis of whether there was a CRF documenting onset of a microbiologically determined infection within the 10 days before the onset of cardiotoxicity.

EFS was defined as the time from study enrollment until death, induction failure, secondary malignancy, or relapse, whereas OS was defined as time to death. RR was defined as the time from enrollment to relapse, secondary malignancy, or induction failure, where deaths without these events were considered competing events. NRM was defined as time from enrollment to death, where relapse, secondary malignancy, or induction failure were considered competing events. Patients were censored at last contact. Compliance at each reporting period was dichotomized based on whether an echo/MUGA was documented.

\section{Covariables}

Patient information, including dates of birth and diagnosis, gender, race, ethnicity, height, weight, initial WBC count, cytogenetic risk classification, treatment arm, and $A E$ reports for bloodstream infection were obtained from the AAML0531 database. Body mass index percentiles for age and sex were computed based on the 2000 Centers for Disease Control and Prevention growth chart data (patients age $\geq 2$ years) and the WHO reference (patients age $<2$ years) and were classified into weight status categories as follows: obese ( $\geq 95$ th percentile), overweight (85th to $<$ 95th percentile), healthy weight (5th to $<85$ th percentile), and underweight ( $<5$ th percentile).

\section{Statistical Analyses}

AAML0531 data used in these analyses were current as of September 30, 2016, with a median follow-up of 6.6 years (range, 0 to 9.8 years) for patients alive at last contact. The 5-year cumulative incidence of cardiotoxicity was presented by covariable categories. The following were enumerated for each reporting period: number of patients who completed protocol-directed echo screenings, number of LVSD events identified by CRF or submitted echo, and number of incident LVSD events.

Log-binomial regression was used to estimate adjusted risk ratios comparing noncompliance by covariables. General estimating equations with an exchangeable correlation matrix were used to account for potential correlation between intra-individual observations. Full follow-up for cardiotoxicity was defined as time from the start of induction I chemotherapy to the first documentation of cardiotoxicity. Patients who did not experience cardiotoxicity were censored at relapse, loss to follow-up, or 5 years after the start of treatment; deaths were considered competing events. Fine and Gray methods ${ }^{16,17}$ were used to compute hazard ratios (HRs) comparing cardiotoxicity incidence by covariables. Initial analyses were performed for the full followup, and then separate assessments were restricted to the on-protocol period, where follow-up ended at the date the 
TABLE 1. Characteristics of the Study Population and the 5-Year Unadjusted Cumulative Incidence of Cardiotoxicity

\begin{tabular}{|c|c|c|c|}
\hline Characteristic & $\begin{array}{c}\text { Covariable } \\
\text { Distribution, } \\
\text { No. (\%) }\end{array}$ & $\begin{array}{l}\text { Cardiotoxicity } \\
\text { Cumulative } \\
\text { Incidence (\%)* }\end{array}$ & $\boldsymbol{P} \dagger$ \\
\hline Age at diagnosis (years) & & & $<.001$ \\
\hline $0-1$ & $207(20.2)$ & 2.2 & \\
\hline $2-10$ & $354(34.6)$ & 12.2 & \\
\hline$\geq 11$ & $461(45.1)$ & 15.6 & \\
\hline Sex & & & .169 \\
\hline Female & $514(50.3)$ & 13.3 & \\
\hline Male & $508(49.7)$ & 10.2 & \\
\hline Race & & & .097 \\
\hline White & $748(73.2)$ & 10.7 & \\
\hline Black & $116(11.3)$ & 17.1 & \\
\hline Other $\ddagger$ & $158(15.5)$ & 12.9 & \\
\hline Ethnicity & & & .209 \\
\hline Hispanic or Latino & 189 (18.5) & 9.2 & \\
\hline Not Hispanic or Latino & $794(77.7)$ & 12.5 & \\
\hline Weight category & & & .474 \\
\hline Underweight & $71(7.0)$ & 7.7 & \\
\hline Normal weight & $603(59.1)$ & 11.5 & \\
\hline Overweight & 152 (14.9) & 11.2 & \\
\hline Obese & 194 (19.0) & 14.7 & \\
\hline Initial WBC count & & & .108 \\
\hline$\leq 100,000 / \mu \mathrm{L}$ & $824(80.6)$ & 12.6 & \\
\hline$>100,000 / \mu \mathrm{L}$ & $198(19.4)$ & 8.7 & \\
\hline Cytogenetic risk group & & & .695 \\
\hline Low $[t(8 ; 21)$ or inv(16)] & 246 (24.9) & (13.0) & \\
\hline Intermediate & 707 (71.6) & 11.5 & \\
\hline High (-5/del5q or -7$)$ & $35(3.5)$ & 8.8 & \\
\hline $\begin{array}{l}\text { Randomized treatment } \\
\text { arm }\end{array}$ & & & .545 \\
\hline
\end{tabular}

\begin{tabular}{lll}
\hline Standard & $511(50.0)$ & 11.2 \\
\hline Standard plus GMTZ & $511(50.0)$ & 12.5
\end{tabular}

Abbreviations: GMTZ, gemtuzumab ozogamicin.

${ }^{*}$ Deaths were considered competing risks in the computation of cumulative incidence.

$\dagger P$ value for the unadjusted comparison of cardiotoxicity occurrence by level of covariable.

$\ddagger$ Asian, American Indian, Alaska Native, Native Hawaiian or other Pacific Islander, those documented as Other race, and those documented as Unknown race.

patient went off protocol. Subanalyses were also performed in which on-protocol incident LVSD in the presence and absence of comorbid infection was evaluated as two separate outcomes, with each being considered a competing event in the assessment of the other. Analyses were also performed to evaluate the occurrence of cardiotoxicity during the off-protocol period among patients who completed the planned protocol therapy; follow-up began once the patient went off protocol, patients who did not experience cardiotoxicity were censored at relapse or last contact, and deaths were considered competing events.

Cox regressions were used to estimate adjusted HR comparing EFS and OS in patients with LVSD (separately for infection-associated or not infection-associated events) relative to patients with no LVSD. Competing risk regressions were used to estimate the subgroup HR comparing RR and NRM by LVSD status. In evaluations of treatment outcomes, LVSD was treated as a time-varying exposure introduced on the day of first occurrence. Cox model-predicted OS, EFS, RR, and NRM curves were presented. Primary analyses considered LVSD regardless of timing; a subanalysis included only on-protocol LVSD. Data for patients with an incident infection-associated cardiac event during the onprotocol period were reviewed for subsequent events that were not infection associated and were reclassified as such in sensitivity analyses. Analyses were performed using SAS 9.3 (SAS Institute, Cary, NC) and STATA 14 (StataCorp, College Station, TX).

\section{RESULTS}

\section{Patient Characteristics}

A total of 1,022 patients with $A M L$ were included in these analyses. Patients were predominantly younger than 10 years of age (55\%), white (73\%), not Hispanic (78\%), and a healthy weight (59\%), with intermediate cytogenetic risk (69\%; Table 1).

\section{Cardiotoxicity Reporting}

Overall compliance with echo evaluations was modest, with $54 \%$ of reporting periods having documentation of completed echoes (Table 2). During the on-protocol period, compliance varied by treatment course; the highest compliance rates were during induction I (85.6\%), intensification I (80.3\%), and intensification III (70.7\%).

Noncompliance was significantly lower during the onprotocol period compared with the off-protocol period (risk ratio, $0.75 ; 95 \% \mathrm{Cl}, 0.71$ to 0.80 ) and during courses with a microbiologically documented bloodstream infection (risk ratio, $0.81 ; 95 \% \mathrm{Cl}, 0.74$ to 0.89 ). Noncompliance did not differ by age, gender, race, ethnicity, weight group, AML risk classification, initial WBC count, or treatment arm (Data Supplement).

Cardiotoxicity increased over on-protocol therapy, with 15 patients $(1.5 \%)$ experiencing toxicity in induction I and 88 (8.6\%) experiencing toxicity by the end of on-protocol treatment. The cumulative incidence of cardiotoxicity over the 5-year follow-up was $12 \%(n=124) ; 25 \%$ of incident events were infection associated, $71 \%(n=88)$ were first documented during on-protocol therapy, and 


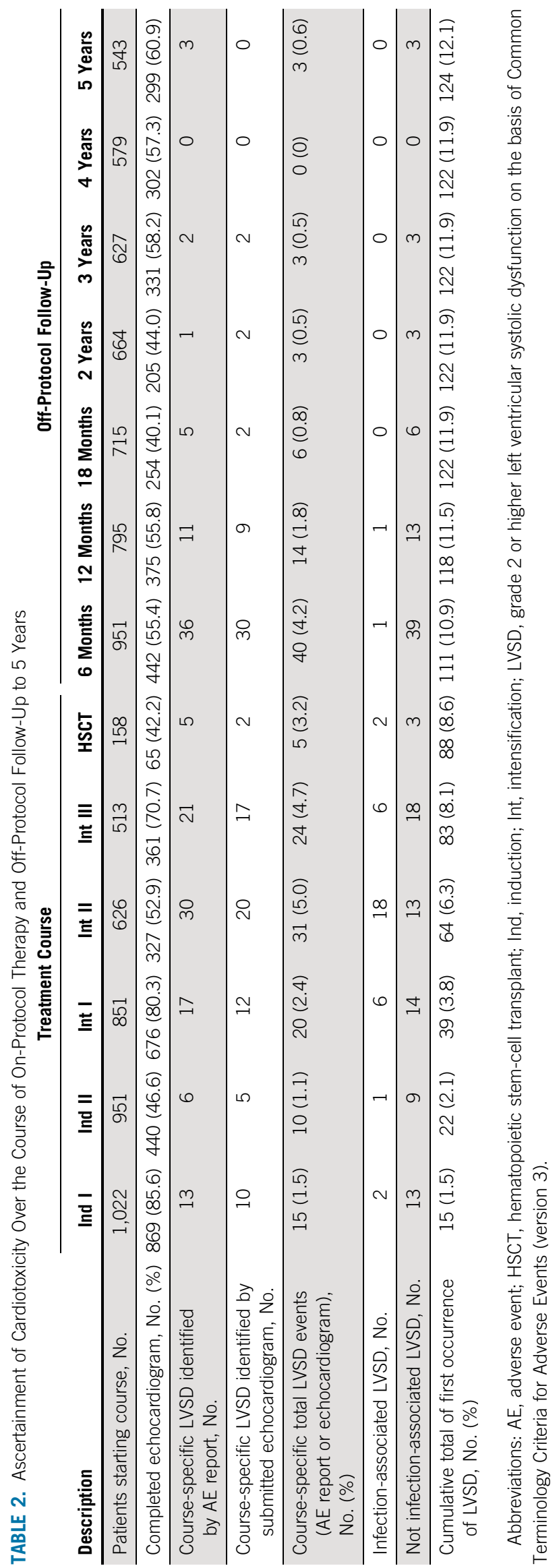


TABLE 3. Multivariable-Adjusted Comparisons of the Occurrence of Cardiotoxicity by Covariables During Overall Follow-Up and Separately for the OnProtocol and Off-Protocol Periods

\begin{tabular}{|c|c|c|c|c|c|}
\hline \multirow[b]{2}{*}{ Characteristic } & \multicolumn{2}{|r|}{ Overall } & \multirow{2}{*}{$\begin{array}{l}\text { On Protocol } \\
\text { HR }(95 \% \mathrm{CI})\end{array}$} & \multicolumn{2}{|c|}{ Off Protocol* } \\
\hline & No.† & HR (95\% Cl) & & No. $\ddagger$ & HR $(95 \% \mathrm{Cl})$ \\
\hline \multicolumn{6}{|l|}{ Age at diagnosis (years) } \\
\hline $2-10$ & 334 & Reference & Reference & 223 & Reference \\
\hline$\geq 11$ & 426 & 1.30 (0.86 to 1.97$)$ & 1.37 (0.85 to 2.21$)$ & 241 & 1.37 (0.47 to 3.97 ) \\
\hline \multicolumn{6}{|l|}{ Sex } \\
\hline Female & 470 & 1.35 (0.92 to 1.99 ) & 1.30 (0.84 to 2.02) & 295 & 2.66 (0.91 to 7.83$)$ \\
\hline Male & 479 & Reference & Reference & 287 & Reference \\
\hline \multicolumn{6}{|l|}{ Race } \\
\hline White & 713 & Reference & Reference & 437 & Reference \\
\hline Hispanic or Latino & 178 & 0.75 (0.43 to 1.31$)$ & $0.63(0.32$ to 1.25$)$ & 105 & 0.78 (0.16 to 3.70$)$ \\
\hline Not Hispanic or Latino & 771 & Reference & Reference & 477 & Reference \\
\hline \multicolumn{6}{|l|}{ Weight category } \\
\hline Underweight & 66 & 0.73 (0.29 to 1.84 ) & 0.55 (0.17 to 1.78$)$ & 46 & 5.26 (1.23 to 22.5$) \S$ \\
\hline Normal weight & 559 & Reference & Reference & 347 & Reference \\
\hline Overweight/obese & 324 & 1.17 (0.78 to 1.76$)$ & 1.04 (0.66 to 1.66$)$ & 189 & 1.85 (0.61 to 5.62) \\
\hline \multicolumn{6}{|l|}{ Cytogenetic risk group } \\
\hline Low $[t(8 ; 21)$ or inv(16)] & 239 & Reference & Reference & 164 & Reference \\
\hline Intermediate & 676 & 1.16 (0.75 to 1.80$)$ & 1.03 (0.64 to 1.66$)$ & 401 & $1.90(0.56$ to 6.44$)$ \\
\hline Standard & 476 & Reference & Reference & 287 & Reference \\
\hline Standard plus GMTZ & 473 & 0.98 (0.67 to 1.44$)$ & $0.82(0.53$ to 1.27$)$ & 295 & $0.39(0.13$ to 1.14$)$ \\
\hline \multicolumn{6}{|c|}{ Microbiologically documented bloodstream infection during treatment } \\
\hline Yes & 683 & $1.66(1.00$ to 2.75$) \S$ & 1.90 (0.98 to 3.71$)$ & & NA \\
\hline No & 266 & Reference & Reference & & \\
\hline \multicolumn{6}{|c|}{ LVSD during the on-protocol period } \\
\hline Yes & & NA & NA & 46 & 12.1 (4.22 to 34.8$) \S$ \\
\hline No & & & & 536 & Reference \\
\hline
\end{tabular}

Abbreviations: GMTZ, gemtuzumab ozogamicin; HR, hazard ratio; LVSD, left ventricular systolic dysfunction; NA, not applicable; NE, not estimable, too few cases of cardiotoxicity among high-risk patients (on the basis of cytogenetics) during off-protocol follow-up.

*Assessment of off-protocol cardiotoxicity was restricted to patients who completed planned protocol therapy.

†Number of patients included in the overall and on-protocol analyses.

$\ddagger$ Number of patients included in the off-protocol analyses.

$\S P$ value $<.05$.

\|Asian, American Indian, Alaska Native, Native Hawaiian or other Pacific Islander, those documented as Other race, and those documented as Unknown race. 
TABLE 4. Multivariable-Adjusted Comparisons of the Occurrence of On-Protocol Cardiotoxicity, Separately for Incident Events Associated With Infection and Not Associated With Infection

\begin{tabular}{|c|c|c|c|}
\hline & & $\begin{array}{c}\text { Infection-Associated } \\
\text { LVSD }\end{array}$ & $\begin{array}{l}\text { Not Infection- } \\
\text { Associated LVSD }\end{array}$ \\
\hline Characteristic & No.* & HR $(95 \% \mathrm{Cl})$ & HR (95\% Cl) \\
\hline \multicolumn{4}{|l|}{ Age at diagnosis (years) $\dagger$} \\
\hline $0-2$ & 242 & 0.12 (0.02 to 0.95$) \ddagger$ & 0.23 (0.07 to 0.80$)$ \\
\hline $3-10$ & 281 & Reference & Reference \\
\hline$\geq 11$ & 426 & 1.13 (0.50 to 2.55$)$ & 1.29 (0.71 to 2.36$)$ \\
\hline \multicolumn{4}{|l|}{ Sex } \\
\hline Female & 479 & 1.76 (0.81 to 3.83$)$ & 1.09 (0.64 to 1.86$)$ \\
\hline Male & 470 & Reference & Reference \\
\hline \multicolumn{4}{|l|}{ Race } \\
\hline White & 713 & Reference & Reference \\
\hline Black & 109 & 3.31 (1.39 to 7.86$) \ddagger$ & 1.63 (0.80 to 3.31$)$ \\
\hline Other§ & 127 & 0.99 (0.29 to 3.43) & 0.74 (0.29 to 1.90$)$ \\
\hline \multicolumn{4}{|l|}{ Ethnicity } \\
\hline Hispanic or Latino & 178 & 0.69 (0.20 to 2.35$)$ & 0.65 (0.29 to 1.46$)$ \\
\hline Not Hispanic or Latino & 771 & Reference & Reference \\
\hline \multicolumn{4}{|l|}{ Weight category } \\
\hline Underweight & 66 & $1.30(0.29$ to 5.78$)$ & 0.31 (0.04 to 2.27) \\
\hline Normal weight & 559 & Reference & Reference \\
\hline Overweight/obese & 324 & 0.78 (0.33 to 1.82$)$ & 1.17 (0.67 to 2.02 ) \\
\hline \multicolumn{4}{|l|}{ Cytogenetic risk group } \\
\hline Low $[t(8 ; 21)$ or inv(16)] & 239 & Reference & Reference \\
\hline Intermediate & 676 & 1.04 (0.46 to 2.35$)$ & 1.00 (0.56 to 1.79$)$ \\
\hline High (-5/del5q or -7$)$ & 34 & 1.59 (0.20 to 13.0$)$ & 0.70 (0.09 to 5.29$)$ \\
\hline \multicolumn{4}{|l|}{ Initial WBC count } \\
\hline$\leq 100,000 / \mu \mathrm{L}$ & 771 & Reference & Reference \\
\hline$>100,000 / \mu \mathrm{L}$ & 178 & 0.99 (0.37 to 2.61) & 0.45 (0.18 to 1.15$)$ \\
\hline \multicolumn{4}{|l|}{ Randomized treatment arm } \\
\hline Standard & 476 & Reference & Reference \\
\hline Standard plus GMTZ & 473 & 1.45 (0.68 to 3.13$)$ & 0.61 (0.36 to 1.06$)$ \\
\hline
\end{tabular}

Abbreviations: GMTZ, gemtuzumab ozogamicin; HR, hazard ratio; LVSD, left ventricular systolic dysfunction.

${ }^{*}$ No. of patients included in the on-protocol analyses.

$\dagger$ Age categories modified because of too few patients with infection-associated LVSD in the 0-to-1-year-old age group.

$\ddagger P<.05$.

$\S$ Asian, American Indian, Alaska Native, Native Hawaiian or other Pacific Islander, and those documented more generally as Other race.

$29 \%$ ( $n=26$ ) were first documented during off-protocol follow-up. Median time to cardiotoxicity was 4.3 months (interquartile range, 3.1 to 5.9). Approximately $61 \%$ of incident LVSD was first documented as grade 2, and $39 \%$ as grade 3 or higher (Data Supplement). Of patients initially documented as experiencing grade 2 LVSD, 18 (24\%) had a subsequent report of higher grade LVSD. The prevalence of cardiotoxicity was variable across treatment courses, ranging from $1.1 \%$ to $5.0 \%$, with intensification II exhibiting the highest prevalence.

\section{Risk Factor Assessment}

Five-year incidence was higher among noninfants and black patients, and was more likely with a bloodstream infection (Table 3). These trends seemed to be driven by toxicities during the on-protocol period, given the consistency of estimates when restricting follow-up to the on-protocol period.

Separate analyses of infection-associated toxicity and toxicity not associated with infection suggested that the higher rate of on-protocol cardiotoxicity among black patients may be specific to infection-associated toxicities (Table 4). Otherwise, there was little difference in risk factors between the two presentations of cardiotoxicity.

Among 622 patients who completed protocol-planned therapy (Data Supplement), 4.8\% $(n=30)$ had cardiotoxicity during off-protocol follow-up. Of those, $46.7 \%$ (n $=14$ ) had cardiotoxicity first documented during onprotocol therapy. Underweight status and LVSD during on-protocol therapy were significantly associated with offprotocol toxicity (Table 3).

\section{Impact of Cardiotoxicity on Treatment Outcomes}

Both 5-year EFS (HR, 1.57; 95\% Cl, 1.16 to 2.14; $P=.004$ ) and OS (HR, 1.59; 95\% Cl, 1.15 to 2.19; $P=.005$ ) were significantly reduced in patients with cardiotoxicity. The impacts on EFS were similar for infection-associated LVSD (36.5\% v 50.0\%; HR, 1.62; 95\% Cl, 0.96 to $2.71 ; P=.069$ ) and LVSD in the absence of infection (32.8\% v50.0\%; HR, $1.55 ; 95 \% \mathrm{Cl}, 1.08$ to $2.23 ; P=.017)$ compared with patients without cardiotoxicity (Table 5; Fig 1). OS for patients with LVSD in the absence of infection was also significantly lower compared with patients without LVSD (45.1\% $\vee 66.0 \%$; HR, 1.72; $95 \% \mathrm{Cl}, 1.19$ to $2.47 ; P=$ .004). The difference in OS was less pronounced for patients with infection-associated LVSD and did not achieve statistical significance (56.7\% v66.0\%; HR, 1.30; $95 \% \mathrm{Cl}$, 0.72 to $2.36 ; 1.98 ; P=.387$ ).

RR was modestly higher among patients who experienced LVSD in the absence of infection compared with those without LVSD (50.1\% v 43.6\%; HR, 1.29; 95\% Cl, 0.86 to 1.92; $P=.217$ ), although the difference was not statistically significant. NRM was higher for both patients with infection-associated LVSD (27.8\% v6.3\%; HR, 4.90; 95\% $\mathrm{Cl}, 1.55$ to $15.5 ; P=.007)$ and those with LVSD in the absence of infection (26.3\% $\vee 6.3 \%$; HR, 2.33; 95\% Cl, 0.97 to $5.63 ; P=0.059$ ). Among patients who completed protocol-planned therapy and experienced LVSD during the off-protocol period $(n=30)$, there were 12 deaths, of which $75 \%$ occurred postrelapse.

In subanalyses limited to on-protocol LVSD, effect estimates for outcomes were essentially unchanged from primary results, with the exception OS, where estimates 
TABLE 5. Multivariable-Adjusted Comparisons of Treatment Outcomes

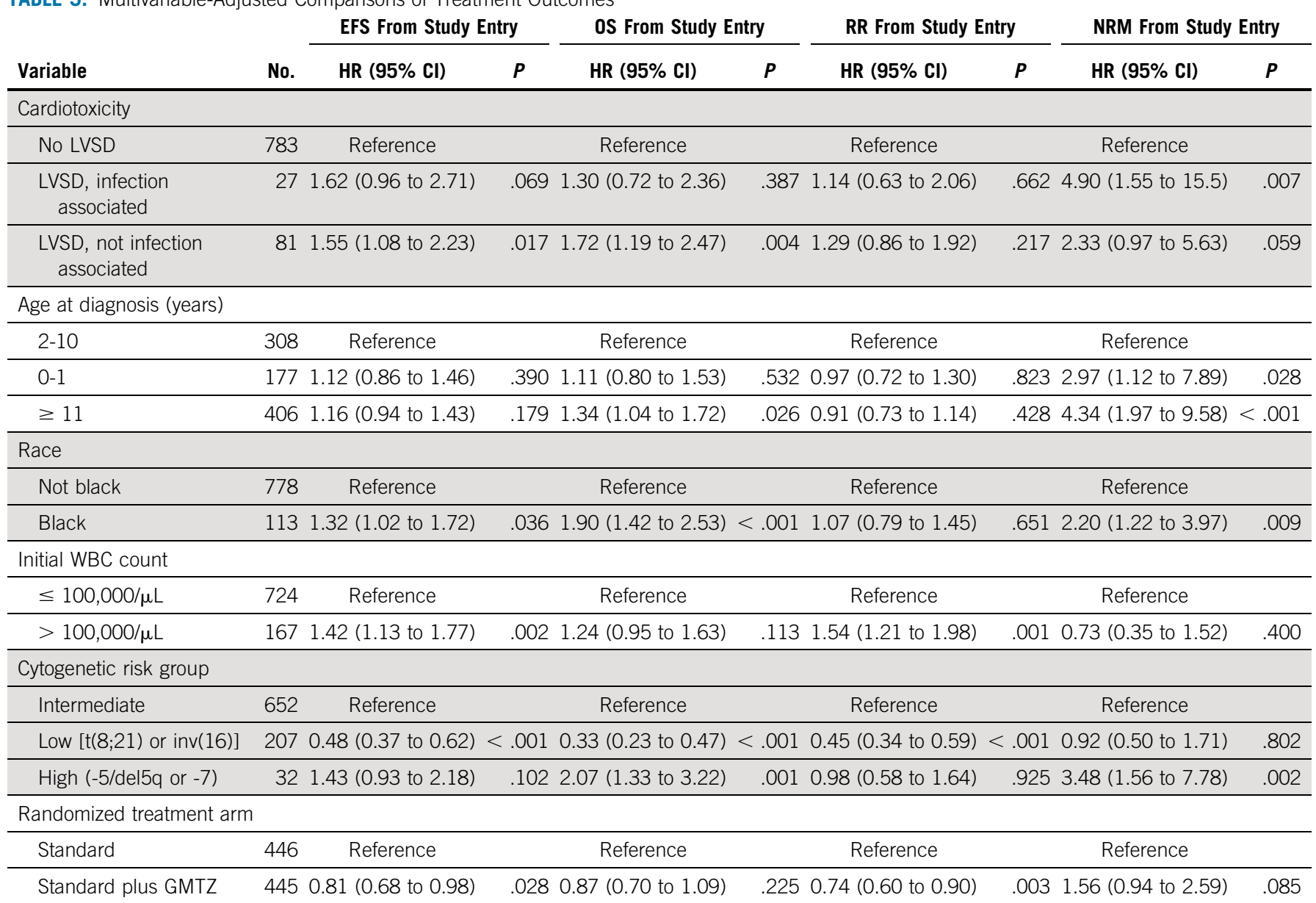

Abbreviations: EFS, event-free survival; GMTZ, gemtuzumab ozogamicin; HR, hazard ratio; LVSD, left ventricular systolic dysfunction; NRM, mortality not related to relapse or induction failure; OS, overall survival; RR, relapse risk.

were slightly attenuated (Data Supplement). Results from sensitivity analyses in which nine patients with infectionassociated incident cardiotoxicity were reclassified because they experienced a subsequent noninfection-related event were also not meaningfully different from the primary analyses (Data Supplement).

\section{DISCUSSION}

Using data from COG AAML0531, we identified novel findings regarding treatment-related cardiotoxicity. Most notably, the occurrence of early cardiotoxicity was associated with statistically significant and clinically meaningful reductions in EFS and OS. The impact of cardiotoxicity on EFS was similar whether it developed in the presence or absence of a bloodstream infection (absolute differences, $13.5 \%$ and $17.2 \%$, respectively), whereas the impact on OS was more profound for cardiotoxicity in the absence of infection than in the presence of infection (absolute differences, $20.7 \%$ and $9.3 \%$, respectively). The relative negative impact of early cardiotoxicity is substantial; the EFS benefit from gemtuzumab ozogamicin (GMTZ) observed in AAML0531 was 6.3\%, which is dramatically less than the observed cardiotoxicity-related decreases in EFS. This dramatic decrease in EFS and resulting decrease in OS underscore the need to understand cardiotoxicity risk factors and to develop effective prevention strategies.

The heterogeneity in the effect of LVSD on OS by comorbid infection status despite similar EFS effects implies that patients who experience cardiotoxicity in the context of infection may be more salvageable on relapse than those who experience noninfection-related cardiotoxicity. Secondary analyses of RR and NRM suggest that the impacts on OS are not entirely the result of an increased risk of induction failure/ relapse. RR was significantly increased with cardiotoxicity in the absence of infection but not with infection-associated cardiotoxicity. In contrast, there was a larger increase in NRM with cardiotoxicity in the presence of infection (HR, 4.90; $95 \% \mathrm{Cl}, 1.55$ to 15.5$)$ than in the absence of infection ( $\mathrm{HR}, 2.33 ; 95 \% \mathrm{Cl}, 0.97$ to 5.63). The discrepancy in NRM may reflect an independent risk associated with bloodstream infections. The difference in the independent predictors of induction failure/relapse and NRM (aside from LVSD) may be useful in identifying subpopulations at risk for the two potentially distinct outcome trajectories. 


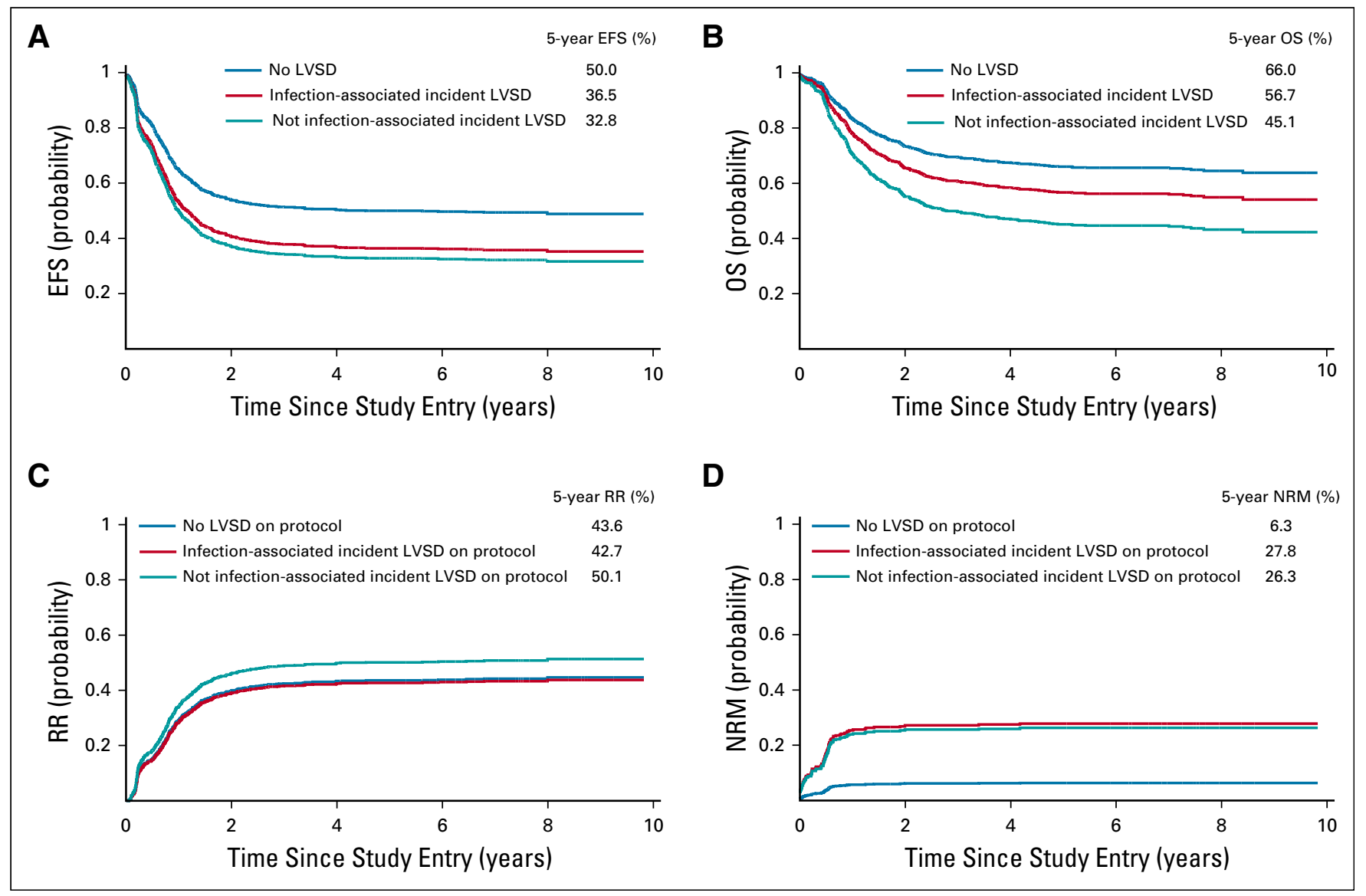

FIG 1. Cox model-predicted curves for (A) event-free survival (EFS), (B) overall survival (OS), (C) relapse risk (RR), and (D) mortality not related induction failure or relapse (NRM). LVSD, left ventricular systolic dysfunction.

Echo monitoring identified cardiotoxicity in $12 \%$ of patients with AML over the course of follow-up, $70 \%$ of whom experienced onset during the on-protocol period. The observed rate of on-protocol cardiotoxicity (8\%) differed from other estimates of early cardiotoxicity. ${ }^{13-15}$ This difference is likely reflective of varied outcome definitions, ${ }^{13-15}$ differing monitoring schedules, ${ }^{13-15}$ and exclusion of cardiomyopathy in the context sepsis ${ }^{13,15}$ or renal failure. ${ }^{13}$

The AAML0531 data represent the first report of coursespecific prevalence of on-treatment cardiotoxicity during pediatric AML therapy. The highest course-specific prevalence occurred in intensification II (cytarabine/mitoxantrone), which seemed to be driven by an increased risk of infectionassociated cardiotoxicity. The prevalence in intensification III, a nonanthracycline course, was similar to that observed in intensification II. This finding argues for evaluating cardiac function after each course of AML chemotherapy, regardless of course-specific anthracycline exposure, as well as additional studies to determine the etiology of this increased risk.

The predictors of incident early cardiotoxicity, including black race and infection, are consistent with those identified for late-onset cardiac dysfunction in childhood cancer survivors. ${ }^{6,9,18-20}$ However, we observed older age at diagnosis to be associated with greater risk for early toxicity, whereas younger age has been historically associated with cardiac dysfunction in longer-term survivors. ${ }^{18-20}$ Chemotherapy dosages in AAML0531 were based on BSA, except for patients with a BSA $<0.6 \mathrm{~m}^{2}$ who were dosed based on weight. Although the chemotherapy delivered to patients $<0.6 \mathrm{~m}^{2}$ is believed to be comparable in intensity, we cannot exclude the possibility that these dosing conversions may have contributed to a lower incidence in infants. Moreover, given that older age is also associated with poorer survival after AML treatment, ${ }^{2,21}$ the discrepancy may indicate a selection bias in the survivor cohorts. This difference in the risk of early versus late cardiotoxicity may also provide some support for the multiple-hit hypothesis of cardiotoxicity, ${ }^{22-24}$ because older pediatric patients may be more likely to have comorbid cardiovascular risk factors. Our finding that LVSD during the on-protocol period was predictive of subsequent off-protocol cardiotoxicity is also consistent with findings of others ${ }^{13-15}$ and with anthracycline-induced toxicity being associated with progressive decline in left ventricular function. ${ }^{6,9}$

Our results should be considered in light of potential limitations. We documented moderate noncompliance with cardiac monitoring over follow-up. We did not identify significant differences in compliance by patient 
characteristics aside from infection, suggesting that the rates of true cardiotoxicity may be underestimated, the magnitude of difference in LVSD occurrence between compared groups may be attenuated, and the heterogeneity by infection status may be somewhat inflated. Compliance was higher in treatment courses for which the echo assessments were documented in the study protocol as Required ( $v$ Recommended), providing observational evidence that mandating surveillance is successful, particularly during treatment. We relied on passive $A E$ reporting as the predominant method of cardiotoxicity ascertainment and did not perform a central echo review, which may have contributed to inconsistencies in LVSD identification and grading. Detailed information on treatment modifications (eg, timing, specific agents, and dose), cardiovascular management after LVSD, and salvage therapies on relapse were not collected. Thus, we were unable to evaluate how cumulative anthracycline exposure, cardiacdirected medications, and relapse regimens may mediate the association between LVSD and EFS/OS. Dexrazoxane use was not captured but was likely used in $<10 \%$ of patients. ${ }^{25,26}$ Thus, our results would generally reflect the occurrence and impact of cardiotoxicity among patients not receiving dexrazoxane. On the basis of the clinically significant impact of early cardiotoxicity on EFS and OS, these data should be included in future clinical trials.

\section{AFFILIATIONS}

${ }^{1}$ The Children's Hospital of Philadelphia, Philadelphia, PA

${ }^{2}$ The Hospital for Sick Children, Toronto, Ontario, Canada

${ }^{3}$ University of Pennsylvania, Philadelphia, PA

${ }^{4}$ Children's Oncology Group, Monrovia, CA

${ }^{5}$ Seattle Children's Hospital, Seattle, WA

${ }^{6}$ AFLAC Cancer and Blood Disorders Center, Emory University/Children's Healthcare of Atlanta, Atlanta, GA

${ }^{7}$ University of Southern California, Los Angeles, CA

${ }^{8}$ Children's Mercy Hospital and Clinics, Kansas City, MO

\section{CORRESPONDING AUTHOR}

Kelly D. Getz, PhD, Perelman School of Medicine, University of Pennsylvania, 423 Guardian Dr, 909 Blockley Hall, Philadelphia, PA 19106; e-mail: getzk@email.chop.edu.

\section{PRIOR PRESENTATION}

Presented in part as poster at the annual meeting of the American Society of Hematology, Atlanta, GA, December 11, 2017.
Despite these limitations, our results offer convincing evidence that early treatment-related cardiotoxicity is associated with significantly decreased EFS and OS. Thus, efforts to improve outcomes for children with AML must include prevention and mitigation of on-protocol cardiotoxicity. Dexrazoxane efficacy analyses from the AAML1031 trial are ongoing. In addition, the next COG frontline $A M L$ trial will evaluate a liposomal anthracycline formulation. Such interventions may not only mitigate the impact of early cardiotoxicity but also late cardiotoxicity, which has been shown by multiple investigators to be the leading cause of late morbidity and nonrelapse-related mortality in pediatric cancer survivors. ${ }^{7,9-12}$ Through a linkage of clinical trial data and administrative data resources, we plan to evaluate the utility of cardiac-directed interventions on the progression and potential resolution of cardiac dysfunction. Finally, additional work is ongoing to discover and validate germline genetic variants that may predispose to anthracycline-associated cardiotoxicity and influence treatment efficacy through drug metabolism or other pharmacogenomics pathways, ${ }^{27-31}$ as well as to investigate the increased risk of cardiotoxicity in black patients. A more complete understanding of the development of cardiotoxicity and effective interventions will inform evidence-based strategies to improve both the cardiovascular and oncologic outcomes for children with cancer.

\section{SUPPORT}

Supported by a National Clinical Trials Network Operations Center Grant (U10CA180886), National Clinical Trials Network Statistics \& Data Center Grant (U10CA180899), and St Baldrick's Foundation.

AUTHORS' DISCLOSURES OF POTENTIAL CONFLICTS OF INTEREST AND DATA AVAILABILITY STATEMENT

Disclosures provided by the authors and data availability statement (if applicable) are available with this article at DOI https://doi.org/10.1200/ JCO.18.00313

\section{AUTHOR CONTRIBUTIONS}

Conception and design: Lillian Sung, Alan Gamis, Richard Aplenc

Administrative support: Leah Sack

Collection and assembly of data: Kelly D. Getz, Robert B. Gerbing, Allison Barz Leahy, Leah Sack, Todd Alonzo, Richard Aplenc

Data analysis and interpretation: Kelly D. Getz, Lillian Sung, Bonnie Ky, Robert B. Gerbing, Kasey J. Leger, William G. Woods, Todd Alonzo, Alan Gamis, Richard Aplenc

Manuscript writing: All authors

Final approval of manuscript: All authors

Accountable for all aspects of the work: All authors

\section{REFERENCES}

1. Faulk K, Gore L, Cooper T: Overview of therapy and strategies for optimizing outcomes in de novo pediatric acute myeloid leukemia. Paediatr Drugs 16:213-227, 2014

2. Gamis AS, Alonzo TA, Meshinchi S, et al: Gemtuzumab ozogamicin in children and adolescents with de novo acute myeloid leukemia improves event-free survival by reducing relapse risk: Results from the randomized phase III Children's Oncology Group trial AAML0531. J Clin Oncol 32:3021-3032, 2014

3. Kaspers GJ, Creutzig U: Pediatric acute myeloid leukemia: International progress and future directions. Leukemia 19:2025-2029, 2005

4. Rubnitz JE: Current management of childhood acute myeloid leukemia. Paediatr Drugs 19:1-10, 2017 
5. Aplenc R, Meshinchi S, Sung L, et al: The addition of bortezomib to standard chemotherapy for pediatric acute myeloid leukemia has increased toxicity without therapeutic benefit: A report from the Children's Oncology Group. Blood 128:899, 2016

6. Curigliano G, Cardinale D, Dent S, et al: Cardiotoxicity of anticancer treatments: Epidemiology, detection, and management. CA Cancer J Clin 66:309-325, 2016

7. Lipshultz SE, Cochran TR, Franco VI, et al: Treatment-related cardiotoxicity in survivors of childhood cancer. Nat Rev Clin Oncol 10:697-710, 2013

8. Lipshultz SE, Franco VI, Cochran TR: Cardiotoxicity in childhood cancer survivors: A problem with long-term consequences in need of early detection and prevention. Pediatr Blood Cancer 60:1395-1396, 2013

9. Chao C, Xu L, Bhatia S, et al: Cardiovascular disease risk profiles in survivors of adolescent and young adult (AYA) cancer: The Kaiser Permanente AYA Cancer Survivors Study. J Clin Oncol 34:1626-1633, 2016

10. Hudson MM, Ness KK, Gurney JG, et al: Clinical ascertainment of health outcomes among adults treated for childhood cancer. JAMA 309:2371-2381, 2013

11. Mertens AC, Liu Q, Neglia JP, et al: Cause-specific late mortality among 5-year survivors of childhood cancer: The Childhood Cancer Survivor Study. J Natl Cancer Inst 100:1368-1379, 2008

12. Mertens AC, Yasui Y, Neglia JP, et al: Late mortality experience in five-year survivors of childhood and adolescent cancer: The Childhood Cancer Survivor Study. J Clin Oncol 19:3163-3172, 2001

13. Creutzig U, Diekamp S, Zimmermann M, et al: Longitudinal evaluation of early and late anthracycline cardiotoxicity in children with AML. Pediatr Blood Cancer 48:651-662, 2007

14. Orgel E, Zung L, Ji L, et al: Early cardiac outcomes following contemporary treatment for childhood acute myeloid leukemia: A North American perspective. Pediatr Blood Cancer 60:1528-1533, 2013

15. Temming P, Qureshi A, Hardt J, et al: Prevalence and predictors of anthracycline cardiotoxicity in children treated for acute myeloid leukaemia: Retrospective cohort study in a single centre in the United Kingdom. Pediatr Blood Cancer 56:625-630, 2011

16. Zanotti-Cavazzoni SL, Hollenberg SM: Cardiac dysfunction in severe sepsis and septic shock. Curr Opin Crit Care 15:392-397, 2009

17. Kohl M, Plischke M, Leffondré K, et al: PSHREG: A SAS macro for proportional and nonproportional subdistribution hazards regression. Comput Methods Programs Biomed 118:218-233, 2015

18. Rose-Felker K, Border WL, Hong BJ, et al: Cardio-oncology related to heart failure: Pediatric considerations for cardiac dysfunction. Heart Fail Clin 13:311-325, 2017

19. Akam-Venkata J, Franco VI, Lipshultz SE: Late cardiotoxicity: Issues for childhood cancer survivors. Curr Treat Options Cardiovasc Med 18:47, 2016

20. Kremer LC, van der Pal HJ, Offringa M, et al: Frequency and risk factors of subclinical cardiotoxicity after anthracycline therapy in children: A systematic review. Ann Oncol 13:819-829, 2002

21. Løhmann DJ, Abrahamsson J, Ha SY, et al: Effect of age and body weight on toxicity and survival in pediatric acute myeloid leukemia: Results from NOPHOAML 2004. Haematologica 101:1359-1367, 2016

22. Sadurska E: Current views on anthracycline cardiotoxicity in childhood cancer survivors. Pediatr Cardiol 36:1112-1119, 2015

23. Minotti G, Menna P, Salvatorelli E, et al: Anthracyclines: Molecular advances and pharmacologic developments in antitumor activity and cardiotoxicity. Pharmacol Rev 56:185-229, 2004

24. Minotti G, Salvatorelli E, Menna P: Pharmacological foundations of cardio-oncology. J Pharmacol Exp Ther 334:2-8, 2010

25. Walker DM, Fisher BT, Seif AE, et al: Dexrazoxane use in pediatric patients with acute lymphoblastic or myeloid leukemia from 1999 and 2009 : Analysis of a national cohort of patients in the Pediatric Health Information Systems database. Pediatr Blood Cancer 60:616-620, 2013

26. Getz KD, Sung L, Leger K, et al: The effect of dexrazoxane exposure on left ventricular function and front line treatment outcomes in patients with acute myeloid leukemia: A report from the Children's Oncology Group. J Clin Oncol 36:10501-10501, 2018 (suppl 15)

27. Linschoten M, Teske AJ, Cramer MJ, et al: Chemotherapy-related cardiac dysfunction: A systematic review of genetic variants modulating individual risk. Circ Genom Precis Med 11:e001753, 2018

28. Armenian SH, Ding Y, Mills G, et al: Genetic susceptibility to anthracycline-related congestive heart failure in survivors of haematopoietic cell transplantation. Br J Haematol 163:205-213, 2013

29. Chang VY, Wang JJ: Pharmacogenetics of chemotherapy-induced cardiotoxicity. Curr Oncol Rep 20:52, 2018

30. Megías-Vericat JE, Montesinos P, Herrero MJ, et al: Impact of ABC single nucleotide polymorphisms upon the efficacy and toxicity of induction chemotherapy in acute myeloid leukemia. Leuk Lymphoma 58:1197-1206, 2017

31. Megías-Vericat JE, Martínez-Cuadrón D, Herrero MJ, et al: Pharmacogenetics of metabolic genes of anthracyclines in acute myeloid leukemia. Curr Drug Metab $19: 55-74,2018$ 
Occurrence of Treatment-Related Cardiotoxicity and Its Impact on Outcomes Among Children Treated in the AAML0531 Clinical Trial: A Report From the Children's Oncology Group

The following represents disclosure information provided by authors of this manuscript. All relationships are considered compensated. Relationships are self-held unless noted. I = Immediate Family Member, Inst = My Institution. Relationships may not relate to the subject matter of this manuscript. For more information about ASCO's conflict of interest policy, please refer to www.asco.org/rwc or ascopubs.org/jco/site/ifc.

\section{Bonnie Ky}

Consulting or Advisory Role: Roche, Bristol-Myers Squibb, Mateon

Therapeutics, Gilead Sciences, Bioinvent, Bristol-Myers Squibb

Research Funding: Pfizer, Roche

Patents, Royalties, Other Intellectual Property: Patent on the use of neuregulin-

$1 \mathrm{~b}$ as a biomarker in heart failure, pending patent application on the use of IgE as

a biomarker of cardiotoxicity

Robert B. Gerbing

Stock and Other Ownership Interests: Pfizer
Kasey Leger

Consulting or Advisory Role: Jazz Pharmaceuticals

Travel, Accommodations, Expenses: Jazz Pharmaceuticals

Alan Gamis

Consulting or Advisory Role: Novartis

Richard Aplenc

Honoraria: Sigma-Tau

Expert Testimony: Wiggin and Dana

Travel, Accommodations, Expenses: Sigma-Tau

No other potential conflicts of interest were reported. 